\title{
Towards a Hybrid Power Cable Health Index for Medium Voltage Power Cable Condition Monitoring
}

\author{
Jose Ignacio Aizpurua \\ Institute for Energy \& \\ Environment \\ University of Strathclyde \\ Glasgow, UK \\ jose.aizpurua@strath.ac.uk
}

\author{
Brian G. Stewart \\ Institute for Energy \& \\ Environment \\ University of Strathclyde \\ Glasgow, UK \\ brian.100.stewart@strath.ac.uk
}

\author{
Martin Kearns \\ EDF Energy \\ East Kilbride, UK \\ martin.kearns@edf-energy.com
}

\author{
Stephen D. J. McArthur \\ Institute for Energy \& \\ Environment \\ University of Strathclyde \\ Glasgow, UK \\ stephen.mcarthur@strath.ac.uk
}

\author{
Nitin Jajware \\ Bruce Power \\ Kincardine, Canada \\ nitin.jajware@brucepower.com
}

\begin{abstract}
Power cables are critical assets for the safe and cost effective operation of the power grid. However, the health assessment of cables is intricate due to its various degradation mechanisms. Motivated by the generation of a consistent cable health index metric, this paper presents a novel hybrid cable health index approach which combines data-driven and physicsof-failure degradation models. Data-driven models are focused on partial discharge tests and physics-of-failure models are focused on cable lifetime estimation based on thermal and electrical stresses. The combination of these models offers an enhanced cable health indicator which supports engineers in the power cable maintenance related decision-making processes.
\end{abstract}

Keywords-cables, health index, prognostics and health management, condition monitoring, data analytics.

\section{INTRODUCTION}

The adoption of prognostics and health management (PHM) strategies is rapidly increasing within the power industry due to the increase of monitored data for different assets such as transformers [1], circuit breakers [2], or cables [3]. PHM strategies focus on monitoring the key degradation parameters of the asset under study so as to implement anomaly detection, diagnostics and prognostics models for condition monitoring and maintenance related decision-making processes.

Condition monitoring and PHM of power cables is intricate due to its various and diverse failure mechanisms including thermal, electrical, mechanical and environmental ageing causes [4]. The potential consequences of cable failures can lead to power outages, which in turn, can result in undesired scenarios such as customer interruptions or accidents. Clearly, power cables are critical assets for the safe and cost-effective operation of the power grid and condition monitoring of power cables is of high relevance for the efficient operation of the power grid.

Monitoring tests and maintenance strategies are different for different cables. For example, more critical cables will be subject to more frequent and exhaustive testing than non-critical cables. Traditionally, separate health assessment tests are performed such as partial discharge or insulation resistance tests in order to diagnose the health of the insulation of power cables $[3,4]$. These separate monitoring tests need to be examined manually by the engineers and they need to reach a consistent conclusion about the health state of the cable. This can potentially result in an error-prone decision making process due to the independent and arduous consideration of the monitoring tests for all the power cables in the grid. In this context, the health index is a pragmatic approach which encapsulates different health assessment models and creates a single consistent health indicator.

The main focus of this paper is on medium voltage (MV) power cables. In order to track the health state of a cable it is critical to identify trending parameters which are consistent over time, ideally until cable failure occurrence. The performance of the cables is influenced by different stressors and ageing mechanisms that will be dependent on the operation context. Thermal, electrical, mechanical and environmental ageing factors affect the cable lifetime. However, there is not always a direct correlation between these parameters and the failure occurrence time. Accordingly, it is necessary to consider different degrading factors altogether so as to evaluate the health of the cable according to different parameters. In this context, this paper presents an initial approach to design a health index framework for cable condition monitoring.

The paper is organized as follows. Section 2 describes the proposed hybrid health index framework. Section 3 develops a case study. Finally, Section 4 draws conclusions and identifies future goals.

\section{Hybrid Power Cable Health Index Framework}

Fig. 1 shows the proposed health index framework which integrates different data sources, physics-based and data-driven cable health assessment models, and finally an evidence 
combination module to combine the designed diagnostics models into a single cable health indicator.

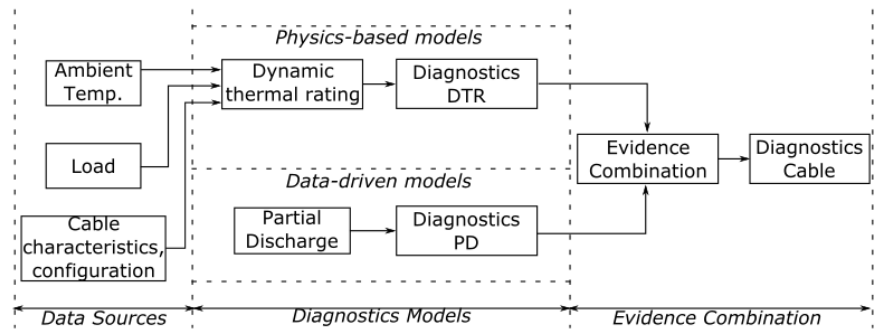

Fig. 1. Hybrid health index framework.

The hybrid health index framework converts input data sources and monitoring tests into a single cable health indicator by using different diagnostics and evidence combination strategies. Note that independent diagnostics modules are deliberately integrated in a modular way so that it is possible to evaluate them independently and altogether. The proposed approach enhances the final decision-making process by providing the engineer with multiple decision points where it is possible to reason about the health of the cable using different subsystem health indexes along with the overall cable health index.

\section{A. Data sources}

The different data sources considered by the hybrid health index framework are the ambient temperature, loading information and cable characteristics as well as cable configuration. The cable characteristics include physical information about the cables such as different layers, their diameters, material, physical location of the cables, and characteristics of the soil. These parameters enable the calculation of the cable conductor temperature and subsequent thermal-stress based degradation.

There are other information sources in the proposed framework which directly reflect the health information of the cables, e.g. partial discharge test results. However, these are considered part of the data-driven diagnostics models due to the diagnostics nature of these tests.

\section{B. Physics-based models}

The main goal of the dynamic thermal rating (DTR) model of the cable is to continuously calculate the cable temperature according to the dynamic operating conditions such as changing load or ambient temperature [5]. The development of a physicsbased DTR model of the cable requires the calculation of a number of parameters dependent on the physical configuration of the cable and the temporal response of the cable to input load profiles.

There are different alternatives to model the cable temperature such as finite element models or equivalent electric circuits [5]. The approach adopted in this paper calculates the cable temperature using an equivalent electrical ladder circuit as shown in Fig. 2 [5]. The thermal response of each layer of the cable (conductor, insulation, sheath, armour, external covering) is modelled using thermal resistances, $T$, capacitances, $Q$, and power losses, $W$.

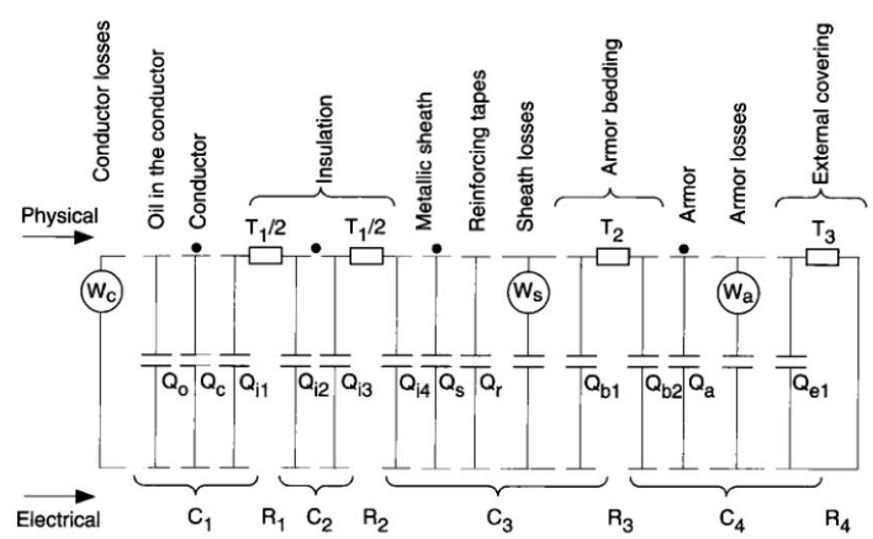

Fig. 2. Cable thermal ladder circuit [5].

Fig. 3 shows the conceptual block diagram so as to calculate the cable conductor temperature based on the electrical circuit in Fig. 2 through different thermo-electric equations.

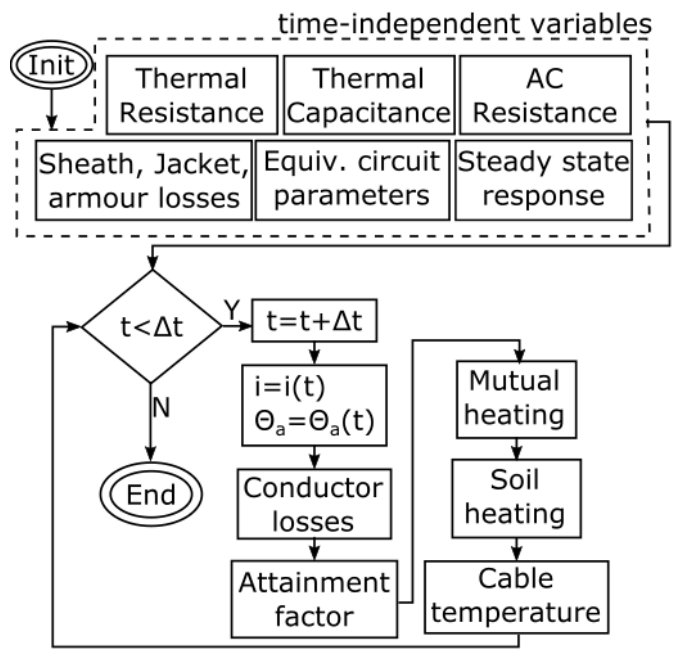

Fig. 3. Cable temperature calculation block diagram.

The time-independent parameters in Fig. 3 are directly calculated from the cable specifications, i.e. thermal resistance, thermal capacitance, AC resistance, losses equivalent circuit parameters and steady state response.

Then, for each time instant, different cable temperature affecting parameters are calculated from the ambient temperature, load and cable characteristics such as conductor losses, attainment factor, mutual heating and soil heating. Finally the final cable temperature is estimated using all the previous parameters. Due to space limits the dynamic thermal rating equations will not be further developed in this work (see [5] for more information and details).

A number of empirical models have been defined to model the effect of electrical and thermal stresses on cable lifetime. For example, the Arrhenius-IPM model relates the cable lifetime with thermal and electrical stresses as follows [4]:

$$
L_{t}=L_{t 0} \exp \left(-B c T\left(E / E_{0}\right)^{-(\eta 0-b c T)}\right)
$$


where $\mathrm{L}_{\mathrm{t}}$ is the lifetime at instant $\mathrm{t}, \mathrm{L}_{\mathrm{t} 0}$ is the lifetime at instant $\mathrm{t}_{0}, B=\Delta w / k$, where $\Delta w$ is the activation energy of the main thermal degradation reaction and $\mathrm{k}$ is the Boltzmann constant, $b$ is a parameter that models the synergism between electrical and thermal stresses, $C_{t}=1 / T_{0}-1 / T$, where $T$ is temperature in Kelvin degrees and $\mathrm{T}_{0}$ is a reference temperature, $\eta_{0}$ models is the voltage endurance coefficient at $T=T_{0}$, and $b$ is a parameter that models the synergism between electrical and thermal stresses.

However, the diagnostics of the cable health state through (1) is hampered because cable temperature estimation is a nontrivial task. It is dependent on a number of factors including the operation environment, cable configuration and applied load among others (cf. Fig. 2 and Fig. 3).

\section{Data-driven models}

There are different cable health monitoring tests that are used to evaluate the health of the cable such as partial discharge (PD), insulation resistance, tan delta, or time-frequency domain response. The adoption of different strategies depends on the monitoring and maintenance strategy of each company.

Note that the proposed health index framework is totally extendible with other information sources. Table I displays different partial discharge levels and associated health for medium voltage (MV) EPR and XLPE cables [6].

TABLE I. PARtial Discharge LeVEls AND Associated Actions.

\begin{tabular}{|c|c|c|}
\hline Discharge Level & MV EPR Cables & MV XLPE Cables \\
\hline \#1: Acceptable & $0-50 \mathrm{pC}$ & $0-250 \mathrm{pC}$ \\
\hline \#2: Minor Concern & $50-120 \mathrm{pC}$ & $250-350 \mathrm{pC}$ \\
\hline \#3: Major Concern & $120-250 \mathrm{pC}$ & $350-500 \mathrm{pC}$ \\
\hline \#4: Action required & $>250 \mathrm{pC}$ & $>500 \mathrm{pC}$ \\
\hline
\end{tabular}

The partial discharge levels in Table I can be converted into PD health index levels as shown in Fig. 4.
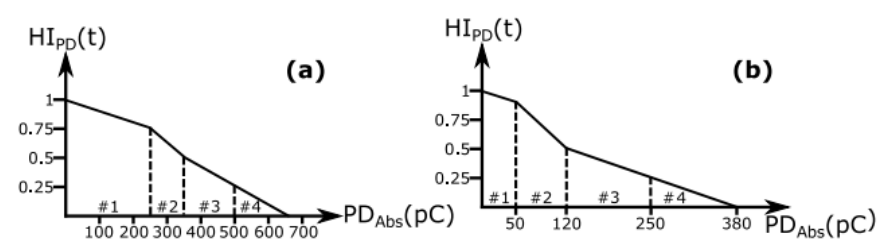

Fig. 4. Partial discharge health index levels inferred from Table I for medium voltage (a) XLPE and (b) EPR cables.

$P D_{a b s}(p C)$ is the measured PD level in $\mathrm{pC}$ units and $H I_{P D}(t)$ is inferred from Table I converting $H I_{P D}(t)$ into $[0,0.25]$ for discharge level \#4, $(0.25,0.5]$ for discharge level \#3, $(0.5,0.75]$ for discharge level \#2, and $(0.75,1]$ for discharge level \#1.

\section{Evidence combination}

The combination of multiple information sources is performed simply by a weighted average procedure:

$$
\mathrm{HI}=\omega_{\text {thermal }} \times \mathrm{HI}_{\text {thermal }}+\omega_{\mathrm{PD}} \times \mathrm{HI}_{\mathrm{PD}}
$$

where $\mathrm{w}_{\text {thermal }}$ and WPD are weights corresponding to the thermal stress and partial discharge and $\mathrm{HI}_{\text {thermal }}$ and $\mathrm{HI}_{\mathrm{PD}}$ are health index values for thermal stress and partial discharge. It has been assumed that the thermal and PD weights are equal for this case study $\left(\mathrm{w}_{\text {thermal }}=0.5, \mathrm{~W}_{\mathrm{PD}}=0.5\right)$. These values should be adapted according to expert knowledge so as to reflect the criticality of the evaluated subsystems.

In order to apply the weighted average combination strategy in (2) all the HI values of different modules need to be normalized between 0 and 1 .

\section{CASE STUDY}

The case study is focused on the health state analysis of a cable with physical and experimental parameters taken from [4, 7]. For the estimation of the lifetime reduction according to thermal stress, a constant ambient temperature of $26^{\circ} \mathrm{C}$ and a constant load of $1 \mathrm{kA}$ are considered. These parameters are repeated for a period of 7000 samples, e.g. which can represent an hourly time-scale which is equal to 292 days approximately. Fig. 5 shows the resulting cable temperature profile over time.

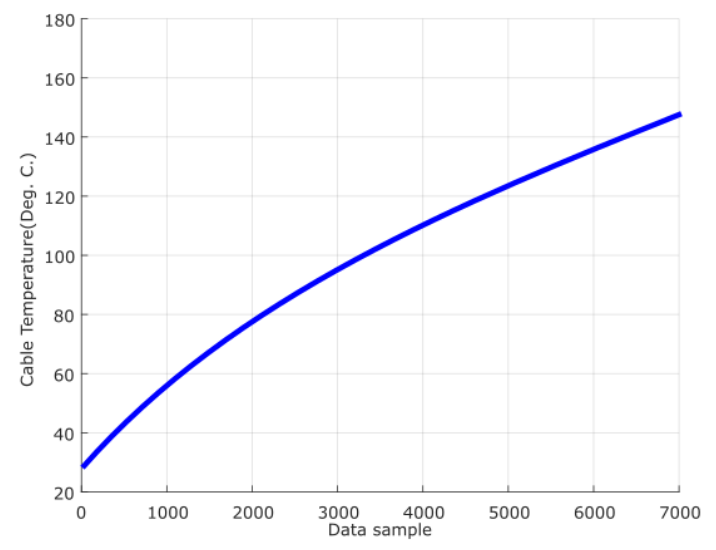

Fig. 5. Cable temperature estimation.

The cable temperature increases exponentially over time with input load, ambient temperature and physical parameters of the cable [5]. With the cable temperatures shown in Fig. 5 the lifetime reduction is calculated through (1) with these parameter values: $\mathrm{L}_{\mathrm{t} 0}=1.93 \times 10^{14}$ hours, $\mathrm{T}_{0}=293 \mathrm{~K}, \mathrm{E}_{0}=5 \mathrm{kV} / \mathrm{mm}$ and $\mathrm{E}=7.2$ $\mathrm{kV} / \mathrm{mm}$ and $\mathrm{b}=4420 \mathrm{~K} . \mathrm{mm} / \mathrm{kV}$. Fig. 6 shows the lifetime reduction over time.

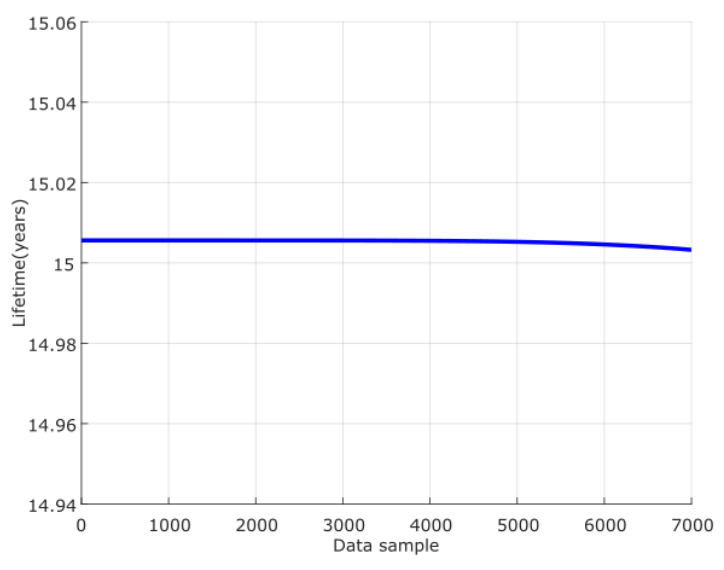

Fig. 6. Cable lifetime estimation. 
It can be seen from Fig. 6 that the lifetime reduction of the cable is very low. This is directly correlated with the cable conductor temperature and adopted parameters $[4,7]$.

Fig. 7a shows examples of some partial discharge measurements over time that could exist on cables and Fig. $7 \mathrm{~b}$ shows the corresponding health index value. By 2011 there was a repair action that lead the cable back to perfect health.
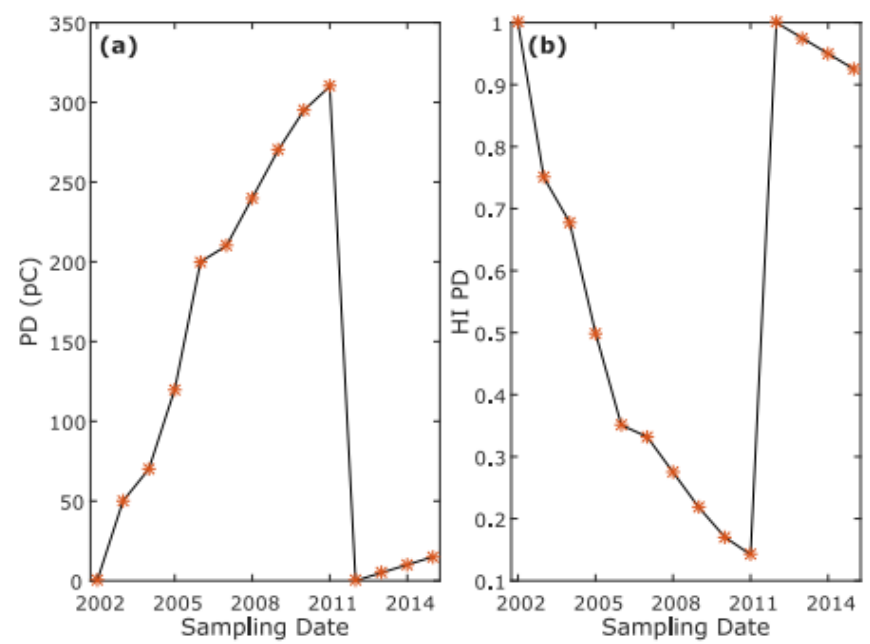

Fig. 7. PD samples and associated health index for EPR cables.

Fig. 8 shows the combined overall health index. The top figure shows the normalized thermal lifetime health index, the middle figure shows the partial discharge health index (cf. Fig. $7 b$ ) and the bottom figure shows the overall cable health index combining both subsystem health indexes through (2).

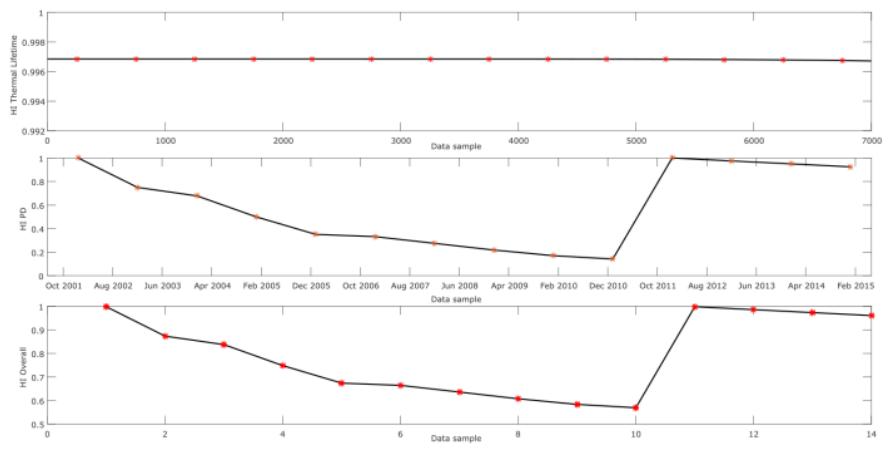

Fig. 8. Combined health index.

Fig. 8 shows the combined overall health index. It can be seen that the thermal stress based lifetime is relatively constant at almost perfect health state, whereas the PD health index constantly decreases until December 2010. After a repair action the PD health index is restored to perfect health. The overall health index effectively captures this trend.
Note that the final amount of health index samples will be limited by the subsystem health index with fewer samples. That is, in this case the thermal stress results were downsampled to match with the PD health samples (cf. Fig. 7).

\section{CONCLUSIONS}

This paper presents a novel hybrid health index framework for cable lifetime management based on data-driven and physics-of-failure models. Independent diagnostics prediction models have been integrated together to demonstrate the generation of a single consistent health index. A case study of the health index framework have been shown to illustrate the applicability of the proposed framework.

The proposed health index framework can assist engineers in the cable condition monitoring process through the identification of early malfunction indicators, diagnostics of different subsystems and estimation of the influence of different operational conditions on the overall cable health.

Future goals will focus on the extension of the proposed health index approach with more diagnostics tests and generating useful technical outcomes for maintenance scheduling.

\section{ACKNOWLEDGMENT}

The work was funded by Bruce Power, EDF Energy, Babcock International and Kinectrics through the Advanced Nuclear Research Centre (ANRC) at the University of Strathclyde.

\section{REFERENCES}

[1] J. I. Aizpurua, S. D. J. McArthur, B. G. Stewart, B. Lambert, J. G. Cross and V. M. Catterson, "Adaptive Power Transformer Lifetime Predictions through Machine Learning \& Uncertainty Modelling in Nuclear Power Plants," IEEE Trans. Industrial Electronics, In Press, 2018.

[2] J. I. Aizpurua, V. M. Catterson, I. F. Abdulhadi and M. S. Garcia, "A Model-Based Hybrid Approach for Circuit Breaker Prognostics Encompassing Dynamic Reliability and Uncertainty," IEEE Transactions on Systems, Man, and Cybernetics: Systems, 48, pp. 1637-1648, 2018.

[3] T.V. Santhosh, V. Gopika, A.K. Ghosh, B.G. Fernandes, "An approach for reliability prediction of instrumentation \& control cables by artificial neural networks and Weibull theory for probabilistic safety assessment of NPPs," Reliability Engineering \& System Safety, 170, pp. 31-44, 2018.

[4] G. Mazzanti, "The combination of electro-thermal stress, load cycling and thermal transients and its effects on the life of high voltage ac cables," IEEE Transactions on Dielectrics and Electrical Insulation, vol. 16, no. 4, pp. 1168-1179, August 2009.

[5] G. Anders, Rating of Electric Power Cables in Unfavorable Thermal Environment, ser. IEEE Press Series on Power Engineering. Wiley, 2005.

[6] "IEEE guide for the measurement of partial discharges in ac electric machinery," IEEE Std 1434-2014 (Revision of IEEE Std 1434-2000), pp. 1-89, Dec 2014.

[7] M. Diaz-Aguilo and F. de Leon, "Adaptive soil model for real-time thermal rating of underground power cables," IET Science, Measurement Technology, vol. 9, no. 6, pp. 654-660, 2015. 